\title{
On the Optimal Synchronization of Oscillator Networks via Sparse Interconnection Graphs
}

\author{
Makan Fardad, Fu Lin, and Mihailo R. Jovanović
}

\begin{abstract}
We consider the problem of synchronizing a given set of oscillators through the design of a conductance network, where the conductance connecting two oscillators models the amount of communication between them. Using optimal control theory, we formulate an optimization problem that addresses the trade-off between synchronization performance and conductance usage. Additionally, we promote the sparsity of the network by penalizing the number of interconnection links. We demonstrate that in the case of identical oscillators the optimization problem is convex and admits formulation as a semidefinite program. For non-identical oscillators that can be considered as perturbations around a central oscillator, we show that it is meaningful to design an optimal conductance network by assuming that all oscillators are identical to the central (average) oscillator. Finally, we derive explicit formulas for the optimal conductance values for some special problems.
\end{abstract}

Index Terms-Convex relaxation, optimization, oscillator synchronization, reweighted $\ell_{1}$ minimization, semidefinite programming, sparse communication architecture.

\section{INTRODUCTION AND MOTIVATION}

Networks of oscillators and their synchronization properties are of interest in a variety of disciplines. For example, Kuramoto oscillators [1] have been the topic of extensive research in the engineering and applied mathematics literature; the recent paper [2] provides an overview of previous work in this area.

For the most part, the literature on oscillator networks deals with finding conditions on oscillator couplings that guarantee their synchronization. These conditions are generally found for an a priori determined interconnection topology of the oscillators, and are often conservative. In the case of Kuramoto oscillators, a problem of particular interest is to characterize the onset of synchronization as a function of the coupling amplitude for a network of oscillators which are all coupled together with identical links.

Two aspects of the synchronization problem, which seem to have not been adequately addressed in the literature to the best of our knowledge, are the issues of interconnection topology design, and, the optimality of the coupling coefficients. This paper attempts to address both issues using tools from optimal control theory and convex optimization.

Financial support from the National Science Foundation under awards CMMI-0927509 and CMMI-0927720 and under CAREER Award CMMI0644793 is gratefully acknowledged.

M. Fardad is with the Department of Electrical Engineering and Computer Science, Syracuse University, NY 13244. F. Lin and M. R. Jovanović are with the Department of Electrical and Computer Engineering, University of Minnesota, Minneapolis, MN 55455. E-mails: makan@syr.edu, fu@umn.edu,mihailo@umn.edu.
We motivate the problem addressed in this paper with the help of a simple example. Consider two LC-oscillator circuits connected by conductance $1 / R$, as in the figure below.

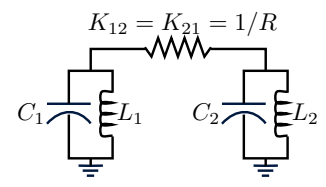

Consider two extreme scenarios:

- $1 / R=0$ : In this case the two oscillators are completely decoupled from each other, and each oscillates at its own resonance frequency $\omega_{i}=\sqrt{1 /\left(L_{i} C_{i}\right)}$.

- $1 / R=\infty$ : In this case the two oscillators are fully coupled. The circuits can be parallel-combined into one oscillator, which oscillates at resonance frequency $\omega_{0} \in\left[\omega_{\min }, \omega_{\max }\right]$ with $\omega_{\min }=\min \left\{\omega_{1}, \omega_{2}\right\}$ and $\omega_{\max }=\max \left\{\omega_{1}, \omega_{2}\right\}$. Thus the network achieves complete synchronization.

In this work, we use the amount of conductance between any two nodes of an oscillator network as a mathematical model for the level of communication between the two oscillators. Therefore, our aim is to synchronize the network in a cost-effective way as far as the overall use of conductance is concerned. In what follows, we consider the synchronization problem for a network of $n$ oscillators. We use the $\mathcal{H}_{2}$ norm to measure the amount of synchronization and also penalize the amount of conductance used. Additionally, a weighted $\ell_{1}$ norm of the conductance matrix is used to penalize the number of interconnection links.

\section{Problem Formulation}

Consider a network of $n$ LC-oscillators, interconnected by a set of conductances. The conductances that connect different oscillators form the edges of a (weighted) graph, with each oscillator connecting a node of the graph to the ground as demonstrated in the figure below.

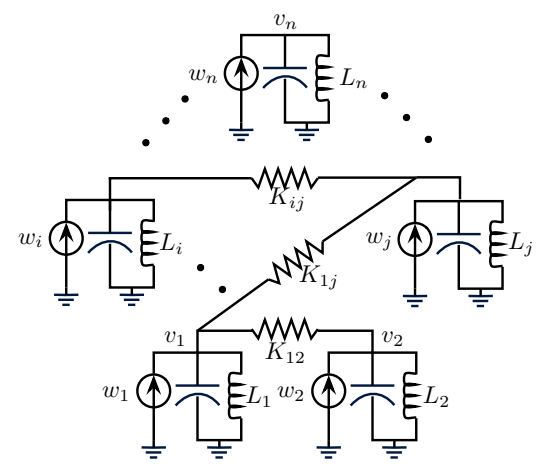


For simplicity, we assume that

$$
C_{i}=1 \quad i=1, \ldots, n,
$$

implying that, when considered in isolation, each oscillator resonates at frequency $\omega_{i}=\sqrt{1 / L_{i}}$.

Let $v$ denote the column vector of node voltages. Then, taking the integral of node voltages $\int_{0}^{t} v$ and the node voltages $v$ as state variables, it is not difficult to show that the dynamics of the entire network can be described by

$$
\dot{\psi}=\left[\begin{array}{cc}
0 & I \\
-H & -K
\end{array}\right] \psi+\left[\begin{array}{c}
0 \\
w
\end{array}\right],
$$

where $\psi=\left[\begin{array}{ll}\xi^{T} & \dot{\xi}^{T}\end{array}\right]^{T}=\left[\begin{array}{ll}\int_{0}^{t} v^{T} & v^{T}\end{array}\right]^{T}$ is the state vector, $w$ is the vector of injected currents into the nodes, and

$$
H=\operatorname{diag}\left\{1 / L_{i}\right\}, \quad K: \begin{aligned}
& \text { conductance matrix of } \\
& \text { node interconnections. }
\end{aligned}
$$

The conductance matrix $K$ [3] can be thought of as a weighted Laplacian [3], [4], which has the property that $K \succeq 0$ and $K \mathbb{1}=0$, with $\succeq$ denoting the positive semidefiniteness of symmetric matrices and $\mathbb{1}$ denoting the column vector of all ones. We assume that the system's graph is connected, which implies the positive definiteness of the matrix $K$ when it is restricted to the subspace $\mathbb{1}^{\perp}$. The measured variables are the node voltages $v$. The state space description (SS) resembles that in [5].

In broad terms, it is desired to find an 'optimal' (in a sense to be made precise in what follows) matrix $K$ such that

(i) the difference in node voltages $\left|v_{i}-v_{j}\right|$ is kept small for every $i$ and $j$;

(ii) the total amount of conductance used to connect nodes is kept small;

(iii) if possible, the number of links between nodes is kept small.

Objective (i) attempts to synchronize the oscillators by keeping the node voltages close to each other. Objective (ii) tries to maintain a small level of communication between the nodes, as conductances between nodes are used to model the amount of communication Objective (iii) is aimed at obtaining a sparse interconnection topology. We note that objective (iii) is sometimes relaxed in this paper, for example, when a particular interconnection topology is determined a priori and optimal values of conductances are sought within that topology.

System (SS) can be written in state-space form [6]

$$
\begin{aligned}
\dot{\psi} & =A \psi+B_{1} w+B_{2} u, \\
z & =C_{1} \psi+D u, \\
y & =C_{2} \psi,
\end{aligned}
$$

and

$$
u=-K y, \quad K \in \mathcal{L},
$$

where

$$
\begin{aligned}
& A=\left[\begin{array}{cc}
0 & I \\
-H & 0
\end{array}\right], \quad B_{1}=\left[\begin{array}{l}
0 \\
I
\end{array}\right], \quad B_{2}=\left[\begin{array}{l}
0 \\
I
\end{array}\right], \\
& C_{1}=\left[\begin{array}{c}
Q^{1 / 2} \\
0
\end{array}\right], \quad C_{2}=\left[\begin{array}{ll}
0 & I
\end{array}\right], \quad D=\left[\begin{array}{c}
0 \\
R^{1 / 2}
\end{array}\right] .
\end{aligned}
$$

The variable $w$ represents the column vector of exogenous inputs that enter the nodes as currents. The matrices $Q$ and $R$ are positive semidefinite and positive definite, respectively. In this control-theoretic framework the matrix $K$ denotes the static feedback gain, which is subject to the structural constraint of being in the set $\mathcal{L}$ of weighted Laplacian matrices. Upon closing the loop, the above problem can equivalently be written as

$$
\begin{aligned}
\dot{\psi} & =\left(A-B_{2} K C_{2}\right) \psi+B_{1} w, \\
z & =\left[\begin{array}{c}
Q^{1 / 2} \\
-R^{1 / 2} K C_{2}
\end{array}\right] \psi,
\end{aligned}
$$

where it is easy to see that the closed-loop $A$-matrix $A-$ $B_{2} K C_{2}$ has the expression

$$
A_{\mathrm{cl}}=\left[\begin{array}{cc}
0 & I \\
-H & -K
\end{array}\right] \text {. }
$$

We further assume that

$$
Q=\left[\begin{array}{cc}
0 & 0 \\
0 & Q_{2}
\end{array}\right]
$$

$$
Q_{2} \mathbb{1}=0, \quad \zeta^{T} Q_{2} \zeta>0 \quad \text { for all } \zeta \neq 0 \text { s.t. } \zeta^{T} \mathbb{1}=0,
$$

and

$$
R=r I, \quad r>0 .
$$

The conditions on $Q_{2}$ imply that it is a positive definite matrix when restricted to the subspace $1^{\perp}$. To justify the structural assumptions on $Q$, we note that in order to achieve synchronization we are interested in making weighted sums of terms of the form $\left(v_{i}-v_{j}\right)^{2}$ small. Since the state vector is of the form $\left[\int v^{T} v^{T}\right]^{T}$, such an objective corresponds to $Q$ matrices with the zero structure displayed in $(\mathrm{Q})$ and $Q_{2}$ matrices that are positive semidefinite and satisfy $Q_{2} \mathbb{1}=0$. For example, in a system of two oscillators, if it is desired to make $\left(v_{1}-v_{2}\right)^{2}$ small then $Q$ has the structure shown in (Q) with

$$
Q_{2}=\left[\begin{array}{c}
1 \\
-1
\end{array}\right]\left[\begin{array}{ll}
1 & -1
\end{array}\right]=\left[\begin{array}{rr}
1 & -1 \\
-1 & 1
\end{array}\right]
$$

It is important to note that due to the structure of $z$, by minimizing the $\mathcal{H}_{2}$ norm of (CL) we are effectively achieving the first two of our optimal synchronization objectives (i)-(ii) outlined earlier. We next formulate the optimization problem considered in this work.

The optimization problem we address in this paper can be formulated as

$$
\begin{array}{cc}
\operatorname{minimize} & \mathcal{J}_{\gamma}:=\operatorname{trace}\left(P B_{1} B_{1}^{T}\right)+\gamma\|W \circ K\|_{\ell_{1}} \\
\text { subject to } & \left(A-B_{2} K C_{2}\right)^{T} P+P\left(A-B_{2} K C_{2}\right) \\
& =-\left(Q+C_{2}^{T} K^{T} R K C_{2}\right) \\
& K \in \mathcal{L}, \quad P \succeq 0 .
\end{array}
$$

where $K$ is the optimization variable, $\|K\|_{\ell_{1}}=\sum_{i, j}\left|k_{i j}\right|$ is the $\ell_{1}$-norm of $K, W$ is a weighting matrix, o denotes elementwise matrix multiplication, and $\mathcal{L}$ denotes the set of weighted Laplacian matrices. We next elaborate on the purpose of the different components in this formulation. 
In the case of $\gamma=0$ the minimization of

$$
\mathcal{J}:=\mathcal{J}_{0}=\operatorname{trace}\left(P B_{1} B_{1}^{T}\right),
$$

subject to the Lyapunov equation in (OPT), is closely related to the standard $\mathcal{H}_{2}$ optimal control problem [6] for finding the feedback matrix $K$. The condition $K \in \mathcal{L}$ ensures that $K$ is also a legitimate conductance matrix. Furthermore, recently it has been demonstrated that $\ell_{1}$ optimization can often be used as a relaxation for $\ell_{0} /$ cardinality minimization [7], [8], where the $\ell_{0}$ norm $\|K\|_{\ell_{0}}$ of a matrix $K$ gives the number of its nonzero entries. Thus the addition of $\|W \circ K\|_{\ell_{1}}$ to the objective function attempts to penalize the number of nonzero elements of $K$, which in terms of the synchronization problem at hand can be interpreted as the number of communication links. Additionally, the weighting matrix $W$ can be updated via an iterative algorithm in order to make the weighted $\ell_{1}$ norm $\|W \circ K\|_{\ell_{1}}$ a better approximation for $\|K\|_{\ell_{0}}$, [8], [9]. We describe one such algorithm in the next paragraph.

Reference [8] introduces the reweighted $\ell_{1}$ minimization algorithm as a relaxation of $\ell_{0} /$ cardinality minimization problems. This methodology was used in [9] to find sparse optimal controllers for a class of distributed systems. We now state the reweighted $\ell_{1}$ algorithm for the sparse optimal synchronization problem:

\section{Sparsity-promoting reweighted $\ell_{1}$ algorithm}

1. Set the iteration count $\mu$ to zero and form the matrix $W^{(0)}=\left[w_{i j}^{(0)}\right]$ with its $i j$ th entry given by

$$
w_{i j}^{(0)}=1 \text {. }
$$

Choose the positive scalar $\varepsilon$ sufficiently small.

2. Solve the weighted $\ell_{1}$ minimization problem (OPT) with $W:=W^{(\mu)}$ to find the (locally) optimal solution $K^{(\mu)}$.

3. Update the weights,

$$
w_{i j}^{(\mu+1)}=\frac{1}{\left|k_{i j}^{(\mu)}\right|+\varepsilon},
$$

where $k_{i j}^{(\mu)}$ is the $i j$ th element of the matrix $K^{(\mu)}$. Form the matrix $W^{(\mu+1)}=\left[w_{i j}^{(\mu+1)}\right]$.

4. Terminate on convergence. Otherwise, increment $\mu$ and go to Step 2.

Henceforth in this paper we will only address solving the optimization problem (OPT) for a given weighting matrix $W$, which corresponds to Step 2 of the above algorithm.

\section{Simplification of Lyapunov Equation}

We examine the structure of the Lyapunov equation that appears in the optimization problem (OPT),

$$
\left(A-B_{2} K C_{2}\right)^{T} P+P\left(A-B_{2} K C_{2}\right)=-\left(Q+C_{2}^{T} K^{T} R K C_{2}\right) .
$$

Substituting the expressions for $A, B_{2}, C_{2}, Q$, and

$$
P=\left[\begin{array}{cc}
P_{1} & P_{0} \\
P_{0}^{T} & P_{2}
\end{array}\right] \succeq 0,
$$

yields

$$
\begin{array}{r}
{\left[\begin{array}{cc}
0 & I \\
-H & -K
\end{array}\right]^{T}\left[\begin{array}{ll}
P_{1} & P_{0} \\
P_{0}^{T} & P_{2}
\end{array}\right]+\left[\begin{array}{cc}
P_{1} & P_{0} \\
P_{0}^{T} & P_{2}
\end{array}\right]\left[\begin{array}{cc}
0 & I \\
-H & -K
\end{array}\right]} \\
=-\left[\begin{array}{cc}
0 & 0 \\
0 & Q_{2}+r K^{2}
\end{array}\right] .
\end{array}
$$

The condition $P \succeq 0$ implies that $P_{1} \succeq 0$ and $P_{2} \succeq 0$. Rewriting this equation in terms of its underlying components yields

$$
\begin{aligned}
H P_{0}^{T}+P_{0} H & =0 \\
P_{0} K-P_{1}+H P_{2} & =0 \\
K P_{2}+P_{2} K-P_{0}-P_{0}^{T} & =Q_{2}+r K^{2} .
\end{aligned}
$$

Finally, using the block decomposition of $P$ to simplify the objective function in (OPT), we have

$$
\operatorname{trace}\left(P B_{1} B_{1}^{T}\right)=\operatorname{trace}\left(P_{2}\right) .
$$

\section{CASE OF UNIFORM INDUCTANCES: A CONVEX PROBLEM}

In this section we make the following simplifying assumption, which we refer to as 'uniform inductance'.

Assumption Let all inductors have the same value, i.e.,

$$
L_{i}=L_{0}, \quad i=1, \ldots, n,
$$

for some $L_{0}>0$. This implies

$$
H=\left(1 / L_{0}\right) I \text {. }
$$

Note that this assumption is restrictive in that all oscillator circuits now have the same resonance frequency $\omega_{0}=$ $\sqrt{1 / L_{0}}$ (recall that all capacitor values are equal to one). However, the synchronization problem is still meaningful, as it still forces the oscillators to match their amplitudes and phases. More interestingly, the uniform inductance scenario can provide a valuable design platform for the more general case in which different inductor values constitute small deviations from some average value,

$$
L_{i}=L_{0}+\delta L_{i}, \quad i=1, \ldots, n,
$$

with $\left|\delta L_{i}\right| \ll L_{0}$. In this case, once the interconnection topology and link weights have been determined by solving the optimization problem (OPT) under the assumption (UI), a perturbation analysis can be employed to update the conductance matrix to accommodate for the nonuniform inductance values in (NI). See [10], [11] for examples of perturbation methods applied to optimal controller design problems. And in Sec. IV we take a perturbation approach to the synchronization problem for non-uniform conductances that satisfy (NI).

From the uniform inductance assumption (UI) it follows that $H=\left(1 / L_{0}\right) I \succ 0$ commutes with any matrix and therefore the first equation in (LYP) becomes

$$
P_{0}+P_{0}^{T}=0 \text {. }
$$

Hence the last equation in (LYP) simplifies to

$$
K P_{2}+P_{2} K=Q_{2}+r K^{2},
$$


with $P_{2} \succeq 0$. Furthermore, from (TP) it follows that

$$
\operatorname{trace}\left(P B_{1} B_{1}^{T}\right)=\operatorname{trace}\left(P_{2}\right),
$$

and therefore the objective in (OPT) is independent of $P_{0}$ and $P_{1}$.

To simplify the optimization problem further, we state the following useful lemma. Let $\mathcal{A}^{\dagger}$ denote the Moore-Penrose pseudoinverse of $\mathcal{A}$.

Lemma 1: Let $\mathcal{A}$ and $\mathcal{Q}$ be symmetric matrices that satisfy $\mathcal{A} \mathbb{1}=\mathcal{Q} \mathbb{1}=0$, and suppose that $\mathcal{A}$ is a negative definite matrix when restricted to the subspace $\mathbb{1}^{\perp}$. Then the following statements hold.

(i) If $\mathcal{Q}$ is a positive semidefinite matrix when restricted to the subspace $\mathbb{1}^{\perp}$, then among all positive semidefinite solutions of the Lyapunov equation $\mathcal{A}^{T} \mathcal{P}+\mathcal{P} \mathcal{A}=-\mathcal{Q}$, the one with the minimum trace satisfies

$$
\operatorname{trace}(\mathcal{P})=-(1 / 2) \operatorname{trace}\left(\mathcal{Q} \mathcal{A}^{\dagger}\right)
$$

Furthermore, this solution satisfies $\mathcal{P} \mathbb{1}=0$ and is a positive semidefinite matrix when restricted to the subspace $\mathbb{1}^{\perp}$.

(ii) Among all solutions of the Lyapunov equation $\mathcal{A}^{T} \mathcal{P}+$ $\mathcal{P} \mathcal{A}=-\mathcal{Q}$, the one which satisfies $\mathcal{P} \mathbb{1}=0$ has the property that

$$
\operatorname{trace}(\mathcal{P})=-(1 / 2) \operatorname{trace}\left(\mathcal{Q} \mathcal{A}^{\dagger}\right) .
$$

(iii) The identity $\operatorname{trace}\left(\mathcal{Q} \mathcal{A}^{\dagger}\right)=\operatorname{trace}\left(\mathcal{Q}\left(\mathcal{A}-\mathbb{1}^{T} / n\right)^{-1}\right)$ holds, and thus for both cases (i) and (ii),

$$
\operatorname{trace}(\mathcal{P})=-(1 / 2) \operatorname{trace}\left(\mathcal{Q}\left(\mathcal{A}-\mathbb{1}^{T} / n\right)^{-1}\right) .
$$

Proof The proof is based on using a special similarity transformation to eliminate the zero mode from the Lyapunov equation $\mathcal{A}^{T} \mathcal{P}+\mathcal{P} \mathcal{A}=-\mathcal{Q}$; the details are omitted for brevity and will be reported elsewhere.

Remark 1: An important utility of this result is that the new description of $\operatorname{trace}(\mathcal{P})$,

$$
\begin{aligned}
\operatorname{trace}(\mathcal{P}) & =-(1 / 2) \operatorname{trace}\left(\mathcal{Q}\left(\mathcal{A}-\mathbb{1}^{T} / n\right)^{-1}\right) \\
& =-(1 / 2) \operatorname{trace}\left(\mathcal{Q}^{1 / 2}\left(\mathcal{A}-\mathbb{1}^{T} / n\right)^{-1} \mathcal{Q}^{1 / 2}\right),
\end{aligned}
$$

lends itself to the application of semidefinite programing (SDP) methods, as we demonstrate below. This is reminiscent of, and was motivated by, the results in [3].

Applying Lemma 1(i) with $\mathcal{A}=-K$ and $\mathcal{Q}=Q_{2}+r K^{2}$ to the Lyapunov equation (P2), in which $P_{2} \succeq 0$, gives

$$
\begin{aligned}
\operatorname{trace}\left(P_{2}\right)= & (1 / 2) \operatorname{trace}\left(\left(Q_{2}+r K^{2}\right) K^{\dagger}\right) \\
= & (1 / 2) \operatorname{trace}\left(Q_{2}\left(K+\mathbb{1}^{T} / n\right)^{-1}\right. \\
& \left.\quad+r K\left(I-\mathbb{1}^{T} / n\right)\right) \\
= & (1 / 2) \operatorname{trace}\left(Q_{2}^{1 / 2}\left(K+\mathbb{1}^{T} / n\right)^{-1} Q_{2}^{1 / 2}+r K\right) .
\end{aligned}
$$

The details of the simplifications in (TP2) are as follows: Since $K$ is the Laplacian of a connected graph then $K \mathbb{1}=0$ and $K$ is positive definite on $\mathbb{1}^{\perp}$. Also, by assumption $Q_{2} \mathbb{1}=0$ and $Q_{2}$ is positive definite on $\mathbb{1}^{\perp}$. Thus $\left(Q_{2}+r K^{2}\right) \mathbb{1}=0$ and $Q_{2}+r K^{2}$ is positive definite on $\mathbb{1}^{\perp}$. Therefore Lemma 1(i) applies and the first equation follows. In the second equation, $Q \mathbb{1}=0$ and the identities $K^{\dagger}=\left(K+\mathbb{1} \mathbb{1}^{T} / n\right)^{-1}-\mathbb{1}^{T} / n, K^{\dagger} K=I-\mathbb{1}^{T} / n$ are invoked. Finally, the last equation follows from $K \mathbb{1}=0$ and the trace identity $\operatorname{trace}\left(M_{1} M_{2}\right)=\operatorname{trace}\left(M_{2} M_{1}\right)$. In summary, (TP2) gives the minimum trace of $P_{2}$ among all positive semidefinite solutions of the Lyapunov equation (P2).

Recall the optimization problem (OPT), which we restate here for convenience

$$
\begin{array}{ll}
\operatorname{minimize} & \operatorname{trace}\left(P B_{1} B_{1}^{T}\right)+\gamma\|W \circ K\|_{\ell_{1}} \\
\text { subject to } & \left(A-B_{2} K C_{2}\right)^{T} P+P\left(A-B_{2} K C_{2}\right) \\
& =-\left(Q+C_{2}^{T} K^{T} R K C_{2}\right) \\
& K \in \mathcal{L}, \quad P \succeq 0 .
\end{array}
$$

Under the uniform inductance assumption (UI), and using (TP) and (TP2), problem (OPT) can be equivalently formulated as

$$
\begin{array}{r}
\operatorname{minimize} \quad(1 / 2) \operatorname{trace}\left(Q_{2}^{1 / 2}\left(K+\mathbb{1} \mathbb{1}^{T} / n\right)^{-1} Q_{2}^{1 / 2}+r K\right) \\
+\gamma\|W \circ K\|_{\ell_{1}}
\end{array}
$$

subject to $K \in \mathcal{L}$.

$(\mathrm{CVX})$

\section{SDP Formulation}

The following proposition is one of the main results of this work. Let

$$
M=\mathbb{1} \mathbb{1}^{T}-I \sim\left[\begin{array}{lll}
0 & 1 & 1 \\
1 & 0 & 1 \\
1 & 1 & 0
\end{array}\right],
$$

and let $\leq$ denote elementwise inequality when applied to matrices.

Proposition 2: The optimization problem (OPT), under the uniform inductance assumption (UI), is equivalent to the semidefinite program

$$
\begin{array}{ll}
\operatorname{minimize} & (1 / 2) \operatorname{trace}(X+r K)+\gamma \operatorname{trace}\left(\mathbb{1}^{T} Y\right) \\
\text { subject to } & {\left[\begin{array}{cc}
X & Q_{2}^{1 / 2} \\
Q_{2}^{1 / 2} & K+\mathbb{1}^{T} / n
\end{array}\right] \succeq 0} \\
& -Y \leq W \circ K \leq Y \\
& M \circ K \leq 0 \\
& K \mathbb{1}=0,
\end{array}
$$

$\left(\mathrm{CVX}^{\prime}\right)$

where the optimization variables are the symmetric matrices $K$ and $X$, and the elementwise-nonnegative matrix $Y$.

Proof The condition $K \in \mathcal{L}$ in $(\mathrm{CVX})$ is equivalent to

$$
K=K^{T}, \quad M \circ K \leq 0, \quad K \mathbb{1}=0 .
$$

The rest of the proof is similar to that of Prop. 1 in [9] and is omitted for brevity.

We note that the optimal conductance matrix is independent of the inductance matrix $H$ when all inductances have the same value. In other words, the optimal $K$ does not depend on the oscillator parameters when all oscillators are identical. 


\section{Special Case: Uniform All-To-All Coupling}

A problem of particular interest in oscillator synchronization is that of uniform all-to-all coupling [1]. This scenario, although nonsparse, can be easily addressed using the framework developed in this paper. In this case every oscillator is connected to all other oscillators and all couplings have the same magnitude (i.e., all conductances have the same value). This implies a particular structure on $K$, namely

$$
K=k\left(I-\mathbb{1}^{T} / n\right)
$$

where $k$ is a nonnegative scalar.

In the uniform all-to-all coupling problem the structure of $K$ is already determined, thus the sparsity-promoting term $\gamma\|W \circ K\|_{\ell_{1}}$ can be removed from the objective of (CVX). Equivalently, we can consider (CVX) with $\gamma=0$. Clearly, the reweighted $\ell_{1}$ algorithm is unnecessary in this case (as $W$ is not used and does not need to be updated), and the problem simplifies to finding the value of $k$ that minimizes $\mathcal{J}=\operatorname{trace}\left(P_{2}\right)$.

It is easy to show that

$$
K^{\dagger}=(1 / k)\left(I-\mathbb{1}^{T} / n\right) .
$$

Thus from (TP2) it follows that

$$
\begin{aligned}
2 \mathcal{J}= & \operatorname{trace}\left(\left(Q_{2}+r K^{2}\right) K^{\dagger}\right) \\
= & (1 / k) \operatorname{trace}\left(Q_{2}\left(I-\mathbb{1}^{T} / n\right)\right) \\
& \quad+r k \operatorname{trace}\left(\left(I-\mathbb{1}^{T} / n\right)^{3}\right) \\
& =(1 / k) \operatorname{trace}\left(Q_{2}\right)+r k(n-1) .
\end{aligned}
$$

Setting $\partial \mathcal{J} / \partial k=0$ gives $k=\sqrt{\operatorname{trace}\left(Q_{2}\right)} / \sqrt{(n-1) r}$ and therefore the optimal $K$ is given by

$$
K=\sqrt{\frac{\operatorname{trace}\left(Q_{2}\right)}{(n-1) r}}\left(I-\mathbb{1} \mathbb{1}^{T} / n\right) .
$$

Notice that the optimal conductance matrix depends only on the trace of $Q_{2}$ and not its exact structure or its individual entries.

\section{GRAdient of $\mathcal{J}$ AND CASE OF NON-UNIFORM INDUCTANCES}

In this section we first state Proposition 3, which is the main result of this work with regards to the case of nonuniform inductances. We then elaborate on the implications of this proposition.

Let $\gamma=0$ and let the different inductor values $L_{i}$ constitute small deviations from some average value $L_{0}$,

$$
L_{i}=L_{0}+\delta L_{i}, \quad i=1, \ldots, n,
$$

with $\left|\delta L_{i}\right| \ll L_{0}$.

Proposition 3: Consider the optimization problem (OPT) in which $\gamma=0$, or equivalently, consider the problem of minimizing $\mathcal{J}=\operatorname{trace}\left(P B_{1} B_{1}^{T}\right)=\operatorname{trace}\left(P_{2}\right)$ subject to the equations (LYP) and the constraints $K \in \mathcal{L}, P \succeq 0$. Small changes $\delta H$ and $\delta K$ in the inductance and conductance matrices, respectively, around the point $H=\left(1 / L_{0}\right) I$ and $K$, result in small changes $\delta \mathcal{J}=\operatorname{trace}\left(\delta P_{2}\right)$ in the value of the objective function. Then, assuming $P_{2} \mathbb{1}=0$ and $\delta P_{2} \mathbb{1}=0$, we have

$$
\delta \mathcal{J}=(1 / 2) \operatorname{trace}\left(\left(r I-K^{\dagger} Q_{2} K^{\dagger}\right) \delta K\right) .
$$

In particular,

$$
\begin{aligned}
\nabla_{H} \mathcal{J} & =0, \\
\nabla_{K} \mathcal{J} & =(1 / 2)\left(r I-K^{\dagger} Q_{2} K^{\dagger}\right),
\end{aligned}
$$

where the gradients are evaluated at the point $\left(\left(1 / L_{0}\right) I, K\right)$.

Proof The proof is omitted for brevity and will be reported elsewhere.

Remark 2: The utility of Proposition 3 can be explained as follows.

- If the inductances of different oscillators can be considered as small perturbations around some average value $L_{0}$ as in (NI) and thus

$$
H(\varepsilon)=H^{(0)}+\varepsilon H^{(1)}, \quad H^{(0)}=\left(1 / L_{0}\right) I,
$$

then $\nabla_{H} \mathcal{J}=0$ implies that up to first order in $\varepsilon$ the value of the objective function $\mathcal{J}$ remains unaffected by changes in $H$. This shows a degree of insensitivity of the objective value $\mathcal{J}$ to the variation of inductances from their average value $L_{0}$. This effectively implies that an optimal design for the case of uniform inductances, where the uniform value is taken to be equal to the average of the different inductances, serves as a good estimate for the globally optimal solution in the case of nonunifrom inductances.

- The gradient $\nabla_{K} \mathcal{J}=(1 / 2)\left(r I-K^{\dagger} Q_{2} K^{\dagger}\right)$ can be used to compute a descent direction in numerical optimization methods. Furthermore, setting $\nabla_{K} \mathcal{J}=0$ gives

$$
K^{\dagger} Q_{2} K^{\dagger}=r I
$$

as a necessary condition for optimality. Multiplying both sides by $K$ and using $K K^{\dagger}=K^{\dagger} K=I-\mathbb{1}^{T} / n$, $Q \mathbb{1}=0$, results in $K^{2}=Q_{2} / r$. Thus the optimal $K$ is given by

$$
K=Q_{2}^{1 / 2} / \sqrt{r}
$$

\section{Special Case: 2-Oscillator Problem}

In this section we use a simple 2-oscillator example to demonstrate the points made in the above remark. Namely, we show the insensitivity of $\mathcal{J}$ to deviations of inductances from a uniform value, and verify that the optimal $K$ is found from $K=Q_{2}^{1 / 2} / \sqrt{r}$.

Let

$$
\begin{gathered}
Q_{2}=q\left[\begin{array}{c}
1 \\
-1
\end{array}\right]\left[\begin{array}{ll}
1 & -1
\end{array}\right]=q\left[\begin{array}{rr}
1 & -1 \\
-1 & 1
\end{array}\right], \\
K=k\left[\begin{array}{rr}
1 & -1 \\
-1 & 1
\end{array}\right], \\
H^{(0)}=\left(1 / L_{0}\right)\left[\begin{array}{ll}
1 & 0 \\
0 & 1
\end{array}\right], \quad H^{(1)}=\left(1 / L_{0}\right)\left[\begin{array}{rr}
1 & 0 \\
0 & -1
\end{array}\right], \\
\Longrightarrow H(\varepsilon)=\left(1 / L_{0}\right)\left[\begin{array}{cc}
1+\varepsilon & 0 \\
0 & 1-\varepsilon
\end{array}\right],
\end{gathered}
$$


where $q$ and $k$ are positive scalars. Then it can be shown that

$$
\begin{aligned}
& P_{0}(\varepsilon)=\left[\begin{array}{ll}
0 & 0 \\
0 & 0
\end{array}\right]+\varepsilon \frac{q+2 r k^{2}}{4 k^{2} L_{0}}\left[\begin{array}{rr}
0 & 1 \\
-1 & 0
\end{array}\right]+O\left(\varepsilon^{2}\right) \\
& P_{1}(\varepsilon)=\frac{q+2 r k^{2}}{4 k L_{0}}\left[\begin{array}{rr}
1 & -1 \\
-1 & 1
\end{array}\right]+\varepsilon\left[\begin{array}{ll}
0 & 0 \\
0 & 0
\end{array}\right]+O\left(\varepsilon^{2}\right) \\
& P_{2}(\varepsilon)=\frac{q+2 r k^{2}}{4 k}\left[\begin{array}{rr}
1 & -1 \\
-1 & 1
\end{array}\right]+\varepsilon\left[\begin{array}{ll}
0 & 0 \\
0 & 0
\end{array}\right]+O\left(\varepsilon^{2}\right)
\end{aligned}
$$

satisfy the equations (LYP) up to first order in $\varepsilon$.

Now, notice that

$$
\mathcal{J}=\operatorname{trace}\left(P_{2}(\varepsilon)\right)=\frac{q+2 r k^{2}}{2 k}+O\left(\varepsilon^{2}\right),
$$

which lacks an $O(\varepsilon)$ term, as predicted in Proposition 3. This demonstrates an insensitivity to small changes in the values of the inductances from their average value.

Furthermore,

$$
\frac{\partial \mathcal{J}}{\partial k}=\frac{4 r k^{2}-2 q}{4 k^{2}}+O\left(\varepsilon^{2}\right) .
$$

Setting the fraction in the equation above equal to zero, gives the optimal value of $k$ for the case of uniform inductances

$$
k=\sqrt{\frac{q}{2 r}} \quad \Longrightarrow \quad K=\sqrt{\frac{q}{2 r}}\left[\begin{array}{rr}
1 & -1 \\
-1 & 1
\end{array}\right] .
$$

To see that this optimal solution is indeed the same as that predicted by (K0), we note that

$$
Q_{2}^{1 / 2}=\sqrt{\frac{q}{2}}\left[\begin{array}{rr}
1 & -1 \\
-1 & 1
\end{array}\right]
$$

which together with $K=Q_{2}^{1 / 2} / \sqrt{r}$ gives the optimal $K$ found above.

Finally, since for the 2-oscillator case the coupling is necessarily uniform and all-to-all, we expect that the optimal solution should satisfy $K=\left(\sqrt{\operatorname{trace}\left(Q_{2}\right)} / \sqrt{(n-1) r}\right)(I-$ $\mathbb{1} \mathbb{1}^{T} / n$ ), as given by equation (KA). Indeed, we have $n=2$, $\operatorname{trace}\left(Q_{2}\right)=2 q$, and thus

$$
K=\sqrt{\frac{2 q}{r}}\left[\begin{array}{rr}
1 / 2 & -1 / 2 \\
-1 / 2 & 1 / 2
\end{array}\right],
$$

which is again the same as the optimal $K$ found above.

\section{AN EXAMPLE}

In this section we consider $n=7$ identical oscillators and design a sparse conductance matrix using the sparsitypromoting algorithm of Sec.II, with the optimization problem in Step 2 of the algorithm being (CVX').

Let $r=1$ and

$$
Q_{2}=\left[\begin{array}{rrrrrrr}
1 & -1 & 0 & 0 & 0 & 0 & 0 \\
-1 & 2 & -1 & 0 & 0 & 0 & 0 \\
0 & -1 & 2 & -1 & 0 & 0 & 0 \\
0 & 0 & -1 & 2 & -1 & 0 & 0 \\
0 & 0 & 0 & -1 & 2 & -1 & 0 \\
0 & 0 & 0 & 0 & -1 & 2 & -1 \\
0 & 0 & 0 & 0 & 0 & -1 & 1
\end{array}\right] \text {. }
$$

The optimal conductance matrices $K_{\gamma}$, for different values of $\gamma$, are given below. As expected, for $\gamma=0$ we recover

$$
\begin{aligned}
& K_{0}=Q_{2}^{1 / 2} / \sqrt{r} \text {. } \\
& K_{0}=\left[\begin{array}{rrrrrrr}
0.84 & -0.52 & -0.13 & -0.07 & -0.05 & -0.04 & -0.03 \\
-0.52 & 1.23 & -0.46 & -0.11 & -0.06 & -0.04 & -0.04 \\
-0.13 & -0.46 & 1.25 & -0.45 & -0.11 & -0.06 & -0.05 \\
-0.07 & -0.11 & -0.45 & 1.25 & -0.45 & -0.11 & -0.07 \\
-0.05 & -0.06 & -0.11 & -0.45 & 1.25 & -0.46 & -0.13 \\
-0.04 & -0.04 & -0.06 & -0.11 & -0.46 & 1.23 & -0.52 \\
-0.03 & -0.04 & -0.05 & -0.07 & -0.13 & -0.52 & 0.84
\end{array}\right]
\end{aligned}
$$

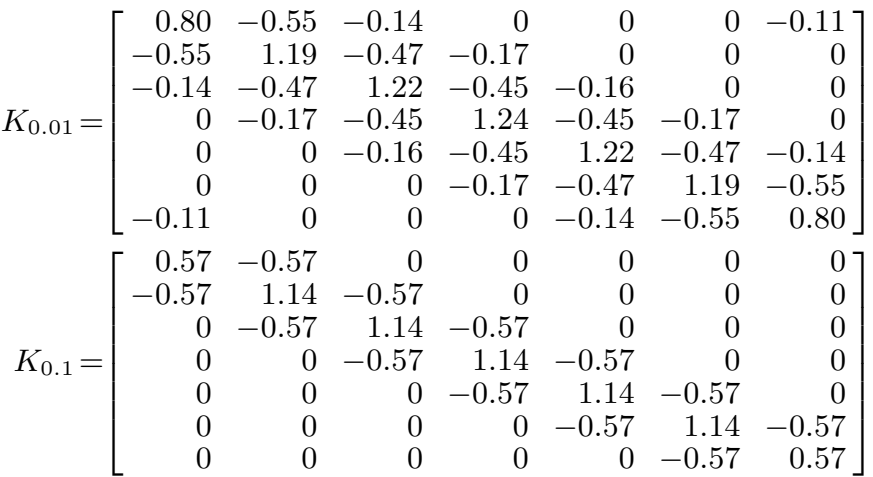

\section{CONCluSions AND FutURE WORK}

We propose an optimization framework for the design of (sparse) interconnection graphs in LC-oscillator synchronization problems. We identify scenarios under which the optimization problem is convex and can be solved efficiently.

Our ultimate goal in this work is to establish a constructive framework for the synchronization of oscillator networks, in which not just the issue of synchronization but the broader questions of optimality and design of interconnection topology can be addressed. One particular class of problems of interest is the Kuramoto oscillator model. It is possible to show that, after applying a sequence of transformations to (CL), the resulting equations closely resemble those of the Kuramoto oscillator. We aim to exploit these similarities in our future work.

\section{REFERENCES}

[1] S. H. Strogatz, "From Kuramoto to Crawford: exploring the onset of synchronization in populations of coupled oscillators," Physica D, vol. 143 , pp. 1-20, 2000.

[2] F. Dorfler and F. Bullo, "On the critical coupling strength for Kuramoto oscillators," in Proceedings of the 2011 American Control Conference, 2011, pp. 3239-3244.

[3] A. Ghosh, S. Boyd, and A. Saberi, "Minimizing the effective resistance of a graph," SIAM Review, vol. 50, no. 1, pp. 37-66, 2008.

[4] F. Dorfler and F. Bullo, "Kron reduction of graphs with applications to electrical networks," SIAM Review, 2011, submitted.

[5] W. Ren, "Synchronization of coupled harmonic oscillators with local interaction," Automatica, vol. 44, pp. 3195-3200, 2008.

[6] K. Zhou, J. Doyle, and K. Glover, Robust and Optimal Control. Prentice Hall, 1996.

[7] E. J. Candès, J. Romberg, and T. Tao, "Stable signal recovery from incomplete and inaccurate measurements," Communications on Pure and Applied Mathematics, vol. 59, no. 8, pp. 1207-1223, 2006.

[8] E. J. Candès, M. B. Wakin, and S. P. Boyd, "Enhancing sparsity by reweighted $\ell_{1}$ minimization," Journal of Fourier Analysis and Applications, vol. 14, pp. 877-905, 2008.

[9] M. Fardad, F. Lin, and M. R. Jovanović, "Sparsity-promoting optimal control for a class of distributed systems," in Proceedings of the 2011 American Control Conference, 2011, pp. 2050-2055.

[10] _ _, "On the optimal design of structured feedback gains for interconnected systems," in Proceedings of the 48th IEEE Conference on Decision and Control, 2009, pp. 978-983.

[11] F. Lin, M. Fardad, and M. R. Jovanović, "Optimal control of vehicular formations with nearest neighbor interactions," to appear in IEEE Transactions on Automatic Control, 2012. 